\title{
DESIGNING CARE PATHWAYS USING SIMULATION MODELING AND MACHINE LEARNING
}

\author{
Mahmoud Elbattah \\ Laboratoire Modélisation, Information et Systèmes \\ Université de Picardie Jules Verne \\ Amiens, FRANCE
}

\author{
Owen Molloy \\ College of Engineering and Informatics \\ National University of Ireland Galway \\ Galway City, IRELAND
}

\author{
Bernard P. Zeigler \\ Department of Electrical and Computer Engineering \\ University of Arizona \\ Tucson, AZ, USA
}

\begin{abstract}
The development of care pathways is increasingly becoming an instrumental artefact towards improving the quality of care and cutting costs. This paper presents a framework that incorporates Simulation Modeling along with Machine Learning (ML) for the purpose of designing pathways and evaluating the return on investment of implementation. The study goes through a use case in relation to elderly healthcare in Ireland, with a particular focus on the hip-fracture care scheme. Initially, unsupervised ML is utilized to extract knowledge from the Irish Hip Fracture Database. Data clustering is specifically applied to learn potential insights pertaining to patient characteristics, care-related factors, and outcomes. Subsequently, the data-driven knowledge is utilized within the process of simulation model development. Generally, the framework is conceived to provide a systematic approach for developing healthcare policies that help optimize the quality and cost of care.
\end{abstract}

\section{INTRODUCTION}

Corollary 1 Public health refers to the art and science of detection and prevention of disease, extending life and promoting health and well-being, through organized efforts and informed choices of society, public and private, communities, and individuals (Wanless 2004).

The above definition illustrates a well-rounded picture of how healthcare services are delivered in complex environments involving many actors and various levels of operation. Further challenges are increasingly raised by population ageing and its impact on health or social policies. All this imperatively calls for developing patient-centred healthcare strategies. In this respect, Modeling and Simulation (M\&S) presents as an effective decision support approach that allows stakeholders to conduct experimental scenarios. Royston (1999) emphasized the significance of computer simulation as an alternative to 'learning by doing' or empirical research methods. In this sense, Pidd (2004) described the model as a vehicle for experimentation, which turns into a tractable surrogate of the actual system being modelled for cheaper and safer manipulation.

The literature is replete with studies that applied M\&S to a multitude of issues pertaining to the healthcare context. This paper discusses the design and evaluation of care pathways (CPs). We present a framework to provide guidance on the design, development, and evaluation of architectures for pathwaysbased coordination of care. The focus is placed on the design and evaluation of architectures that treat 
patients as agents interacting with systems or services coordinated using health information networks and electronic medical records. Success in this direction can contribute to the universal goal of optimising healthcare systems in terms of curbing costs while maintaining or improving care quality. The framework builds upon previous work developed by (Zeigler and Sarjoughian 2013; Zeigler 2016). The basic addition is to include Machine Learning (ML) as an assistive artefact to support the development of simulation models. In particular, we consider the use of unsupervised ML techniques to gain insights into identifying the population at risk, and developing early intervention care schemes. As an example, this paper applies a use case in relation to the hip fracture treatment in Ireland.

\section{BACKGROUND AND METHODOLOGY}

This section provides a basic background that sets the scene for the rest of the paper. Initially, the concept of CPs is overviewed. Subsequently, we present a framework for designing CPs that extends the earlier work by (Zeigler 2016) as elaborated in the ensuing discussion.

\subsection{Care Pathways}

CPs were described as a method for the care management of a well-defined group of patients during a welldefined period of time (De Bleser et al. 2006). Generally speaking, CPs consist of algorithms (usually informally presented as a flow chart with conditional branching) that delineate the overall structure of decision-making for treating a specific medical condition. The aim of CPs is to enhance the quality of care across the continuum by improving risk-adjusted care outcomes, promoting patient safety, increasing patient satisfaction, and optimizing the use of resources (Vanhaecht 2007).

As acknowledged in literature (e.g. Pearson et al. 1995; Every et al. 2000; Vanhaecht et al. 2010), CPs are referenced under a variety of names including Clinical Pathways, Critical Pathways, Clinical Process Models, Integrated Care Pathways, and Care Maps. Further, there is no widely accepted definition, although key characteristics have been extracted from over 200 articles (De Bleser et al. 2006). In light of that concept analysis provided by (De Bleser et al. 2006), an all-inclusive definition of CPs was formulated by the European Pathway Association (EPA 2018) as follows:

Definition 1 A care pathway is a complex intervention for the mutual decision making and organisation of care processes for a well-defined group of patients during a well-defined period.

Since their introduction in the 1990s, CPs have become widespread in healthcare management (Pearson et al. 1995). A comprehensive analysis of 27 studies compared outcomes and costs for hospitals that used CPs with those that do not (Rotter et al. 2010). It appeared that the use of CPs was correlated with benefits in reduction of in-hospital complications, decreased length of stay (LOS), and decreasing hospital costs. Another large-scale study for knee surgery found reduction in LOS and avoidance of treatment complications for hospitals using CPs (Husni et al. 2010). However, not all studies indicate the successful application of CPs. For instance, (Rotter et al. 2010) noted that although the use of pathways tended to improve documentation, poor reporting, nevertheless, prevented the identification of characteristics common to application of successful pathways. (Shi et al. 2008) enumerated eight types of factors that can influence results. They consider factors in terms of three aspects as follows:

- CP Design: Inclusion of all participating disciplines, applicability to intended medical conditions, flexibility incorporated in pathway specification.

- CP Execution: Training of participants, continuous improvement, psychological influences, computer-support.

- CP Evaluation: Consideration of multiple factors. 


\subsection{A Framework for Designing and Evaluating Care Pathways}

Recent interest in computerization of CPs has stimulated a considerable work in treatment of pathways from the perspectives of computer science and software engineering. Zeigler (2016) presented a framework for modeling the architectures of coordinated care. The framework included components that facilitate assessing their interactions, efficacies, and costs in combination. The framework conceived coordination as a layer added to the health information technology (HIT) linking patients with the healthcare system.

Zeigler's view considered CPs as a mathematical system model that is being designed and manipulated to support re-engineering an existing real-world clinical process. This allows for taking a systemsengineering approach in which the system is modelled and simulated before being implemented in reality (i.e. operationalised in a hospital environment). The framework approach takes the following phases:

1. Generate target population of appropriate physical, behavioural and socioeconomic characteristics.

2. Track patients through system with and without coordination of care.

3. Evaluate cost of coordination as addition to total cost.

4. Evaluate improvement in outcome and effect on reduction in total cost.

5. Compute Return on Investment (ROI) $=$ Cost saving per dollar of coordination cost.

The framework was practically applied in a use case to evaluate the ROI of pathway-based intervention in low birthweight pregnancies (Zeigler et al. 2016). The work presented in this paper attempts to extend Zeigler's framework by including a component for incremental learning from data. Healthcare is delivered in data-rich environments where sheer amounts of data are created in patient records at the population and individual levels. ML can therefore present as a key enabling factor to avail of such data repositories to provide insights for cost cutting, care improvement, or identifying the population at risk.

In this regard, our viewpoint is that unsupervised ML can suitably serve the purpose of knowledge elicitation at early stages of problem formulation. Unsupervised techniques (e.g. clustering, rule mining) can be employed during conceptual modelling as an assistive artefact to help conceptualise the structure or behaviour of the healthcare system. For example, data clustering can be utilised to discover significant structures or patterns, which would represent an abstraction of the actual healthcare system under study. This can in turn reflect on the simulation model used to design the architecture of coordinated care. Figure 1 illustrates Zeigler's framework along with the ML component.

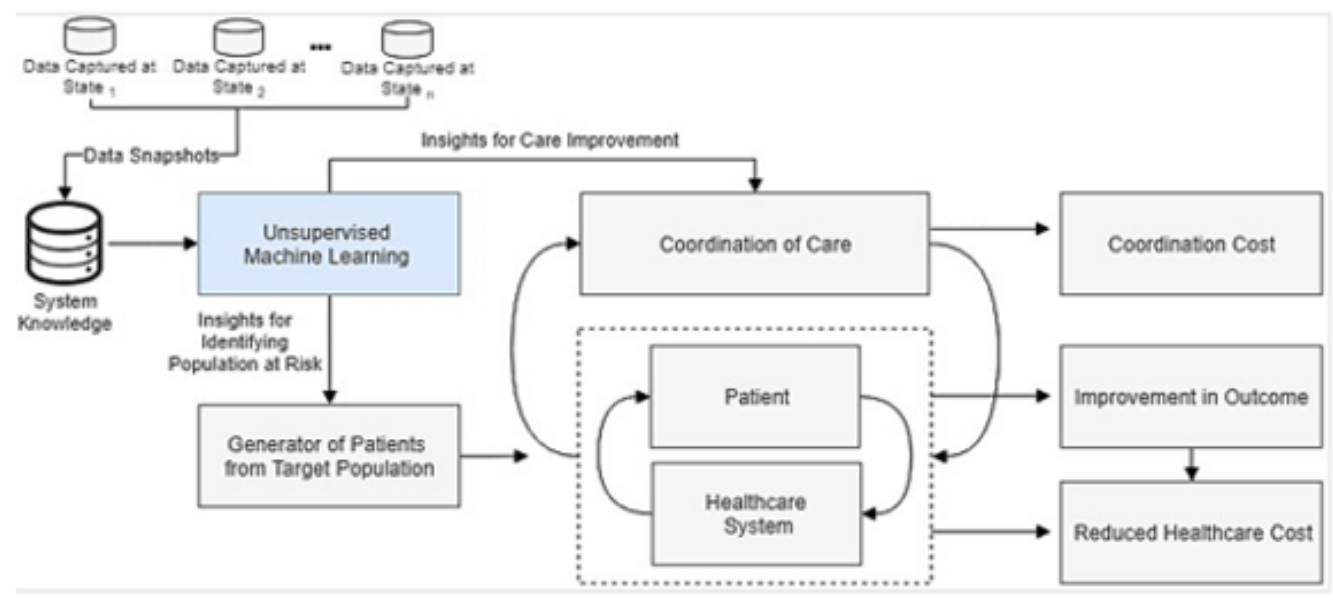

Figure 1: Framework architecture.

A key point addressed by the extended framework is the role of incremental learning. The framework aims at training and fitting ML models on an accumulative basis, rather than a one-off process. The idea of 
incremental learning is predicated on the premise that new conditions (i.e. system states) are being continuously captured in timely snapshots of data and added up to a repository representing the accumulated system knowledge. In this manner, ML models can be iteratively trained to learn about possible updates in the actual system.

\section{EXAMPLE: COORDINATION OF HIP FRACTURE CARE IN IRELAND}

A use case is provided in this section. The following segments elaborate the case setting, and the development of simulation and ML models. The main goal here was to provide a practical scenario where simulation models can be designed or guided in concert with knowledge learned from ML as proposed by the conceptual framework.

\subsection{Overview of the Healthcare System in Ireland}

Simulation studies should start with understanding the actual system, gathering data, and organizing information pertaining to the problem of interest (Law 2007). Likewise, in the ML context the CRISP-DM framework (Shearer 2000), for example, sets the perception of business and data as the initial crucial stage in the process of data mining. In this sense, this section delivers a basic background of the healthcare system in Ireland, and its underpinning components.

Starting with an outlook on the population, the implications of ageing continue to be a matter of considerable concern for care delivery in Ireland. The Health Service Executive (HSE) reported in 2014 that the increase in the number of people over 65 is approaching $20 \mathrm{~K}$ per year (HSE 2014). Population ageing is therefore expected to have profound impacts on a broad range of economic and social areas.

In response to the foreseeable challenges, the healthcare system has been undergoing a substantial reform based on a phased strategy since 2012. The fundamental goal of the reform is to transition the healthcare system towards the integrated delivery of healthcare services. The integrated care approach has been adopted to improve healthcare services regarding accessibility, quality and user satisfaction. As described by (Gröne and Garcia-Barbero 2001), integrated care is a concept that brings together inputs, delivery, management and organisation of services related to diagnosis, treatment, care, rehabilitation and health promotion. Figure 2 sketches the development phases towards realising integrated care.

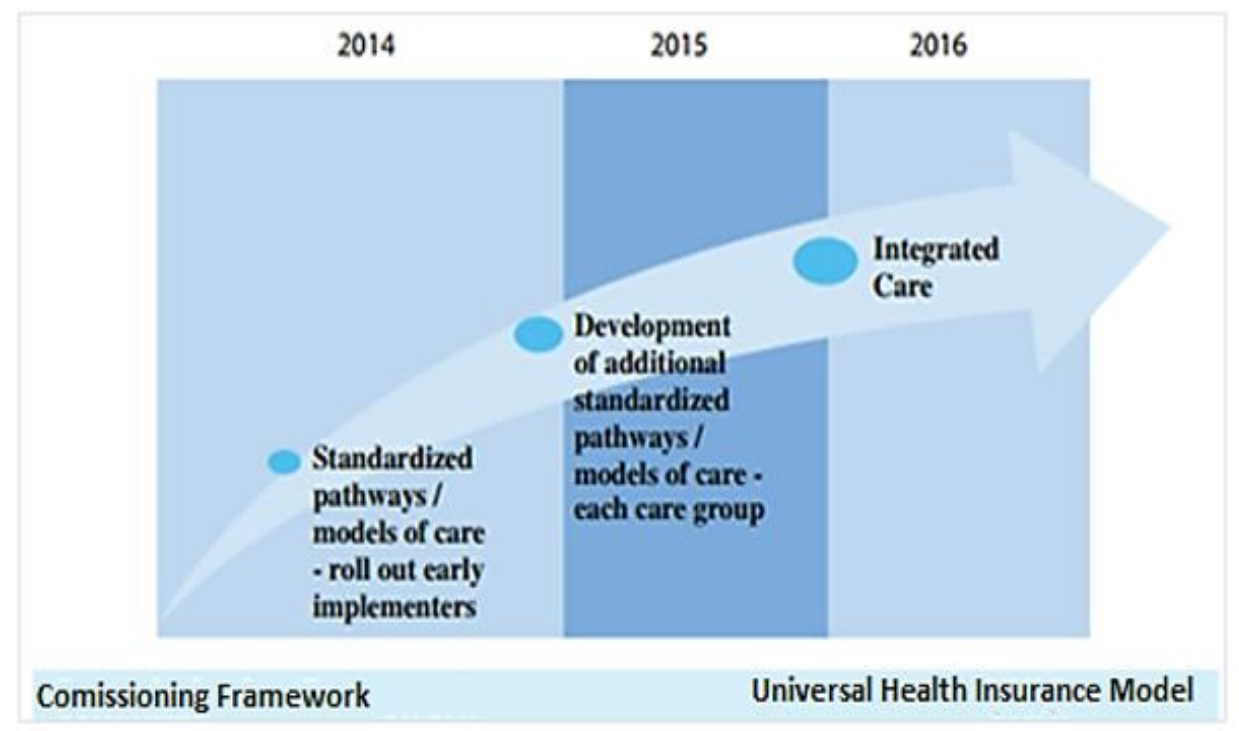

Figure 2: The development phases of healthcare system in Ireland (HSE 2015). 
The transitional arrangements included structuring the Irish healthcare system into nine geographic regions named as Community Health Organisations, commonly referred as $\mathrm{CHO}$. The CHOs can be likened to the Accountable Care Organisations (ACO) in the US healthcare system (Gold 2015). Similar to the ACOs, the establishment of CHOs focuses on providing coordinated care for patients, such that they get the right care at the right time. To put it in more detailed words, the CHOs are aimed to serve as integrated service areas that can deliver better, more integrated and responsive services to people in the most appropriate setting. Figure 3 shows the geographic boundaries of the designated CHOs.

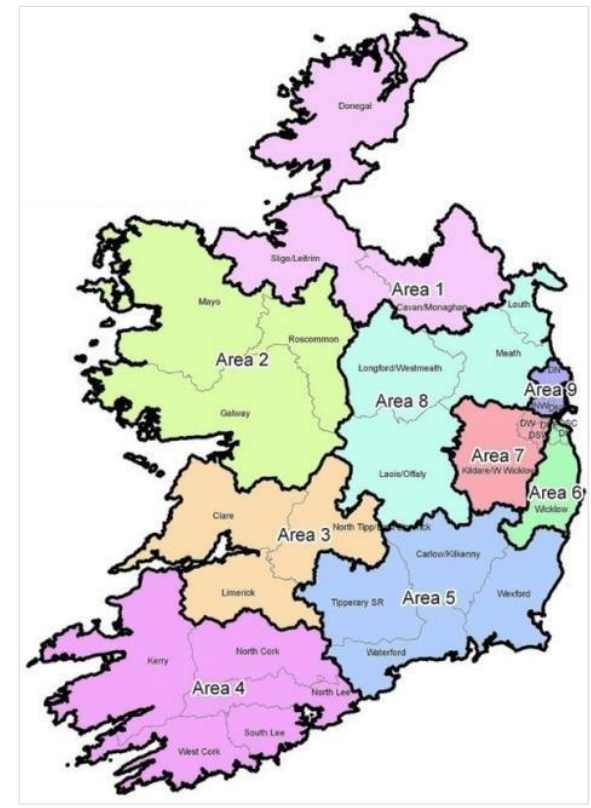

Figure 3: The geographic boundaries of CHOs (HSE 2015).

\subsection{Our Focus: Hip Fracture Care in Ireland}

In this study, we present a use case focusing on developing pathways for hip fracture care in Ireland. In general, hip fractures are a major cause of injuries and morbidity among the elderly. In this regard, numerous studies (e.g. Cooper et al. 1992; Melton 1996) recognized hip fractures to be exponentially increasing with age.

In Ireland, it was reported that around 3K people sustain hip fractures annually (Dodds et al. 2009). Specifically, the rates of the total population (aged 50 years and over) were estimated as 407 and 140 per $100 \mathrm{~K}$ for females and males respectively. Further, the accelerating population ageing could be partly responsible for increasing those rates in the future.

From an economic perspective, hip fractures represent a significant burden on the Irish healthcare system. According to the HSE, hip fractures are considered as one of the most serious injuries that result in lengthy hospital admissions and high costs as well (HSE 2008a). The average LOS was reported as 12.7 days, and more than two-thirds are usually discharged to long-stay care facilities after surgery. Moreover, about $80 \%$ of the patients are over 75 years old, which increases the possibility of being susceptible to further complications (HSE 2008b). In view of that, the incidence of hip fractures represents a major concern to healthcare in Ireland, which calls for the development of effective care instruments (e.g. care pathways) in order to meet the foreseen challenges. 


\subsection{Data Description}

The Irish Hip Fracture Database (IHFD) is the main source of our data. The IHFD repository was developed to serve as the national clinical audit that captures care standards and outcomes for hip-fracture patients in Ireland. The IHFD archives abundant information about the patient's journey from admission to discharge. Specifically, a typical patient record includes 38 data fields about a variety of information including patient characteristics (e.g. gender, age), and care-related factors (e.g. LOS). A thorough explanation of the data fields is available via the official data dictionary (HIPE 2015). We acquired a dataset that covered three years from January 2013 to December 2015 including about 8K records with a specific focus on elderly patients aged 60 and over.

It is worth mentioning a couple of points. First, the dataset was not evenly divided up among the CHOs. It turned out that the CHOs along the eastern side of the country included the largest part of the patient records. Other areas such as $\mathrm{CHO} 1, \mathrm{CHO} 2$, and $\mathrm{CHO} 4$ had relatively less number of records in the dataset. Second, a single patient may have been related to multiple records in case of recurrent fractures. However, we were unable to estimate the proportion of such recurrent cases, whereas records were fully anonymised for the purpose of privacy.

\subsection{Unsupervised Machine Learning: Discovering Patient Clusters}

Clustering was generally defined as the segmentation of a heterogeneous population into a number of more homogeneous subgroups (Aldenderfer and Blashfield 1984). As such, the IHFD records presented a good opportunity to realize the grouping of patients from a data-driven viewpoint. The attempt was to group patients based on the similarity of characteristics, care-related factors, and outcomes. The clusters could help explore the similarities and differences between entities (i.e. patients) that compose the system under study. The following sections briefly summaries the data pre-processing procedures, and clustering experiments. A detailed presentation of those aspects would go beyond the scope of this study.

\subsubsection{Selected Features}

Initially, the clustering model included LOS and age as features. More features could be extracted from the dataset, which are related to quality standards as reported by the British Orthopaedic Association (Darowski 2007). In this respect, two quality measures could be captured from the IHFD dataset as follows:

- All patients should be admitted to an orthopaedic ward within 4 hours of presentation.

- All patients should have surgery within 48 hours of admission.

Our intuition was that those factors could have an influence on care outcomes, and they can therefore serve as candidate features in the clustering model. Both could be derived based on the date/time values of patient arrival, admission and surgery. In this way, two more features were added named as 'Time to Admission (TTA)' and 'Time to Surgery (TTS)'. However, the TTA was subsequently excluded as it contained a significant amount of missing values. The min-max normalisation method was used, where all features were linearly scaled to the $[0,1]$ interval.

\subsubsection{Clustering Experiments}

The clustering model was implemented using the K-Means algorithm with the number of clusters $(\mathrm{K}=2: 5)$. Initially, the quality of clusters was examined based on the within-cluster sum of square (WSS). Initially, it appeared that three or four well-detached clusters that could best separate the patient groups. To further investigate cluster modularity, the clusters were projected using the Principal Component Analysis (PCA). The clusters were transformed into two dimensions with PCA, as plotted in Figure 4. Each sub-figure represents the output of a clustering experiment using a different number of clusters $(\mathrm{K})$. With $\mathrm{K}=2$, the output indicated a promising tendency of clusters, where the data space is obviously separated into two big 
clusters. Similarly, when $\mathrm{K}=3$ the clusters are still well-separated. However, the separation of clusters started to decline when $\mathrm{K}=4$ onwards. Thus, it turned out eventually that there were three clusters that divided the dataset into coherent patient cohorts. The experiments were run using the Azure ML Studio. The general characteristics of clusters are briefly presented in Table 1. The table compares clusters in terms of average age, gender distribution, and other care-related factors (e.g. LOS and TTS).

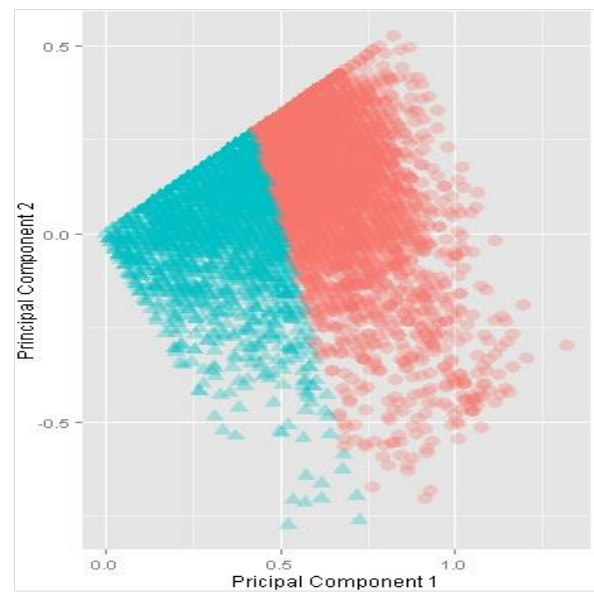

(a) $\mathrm{K}=2$

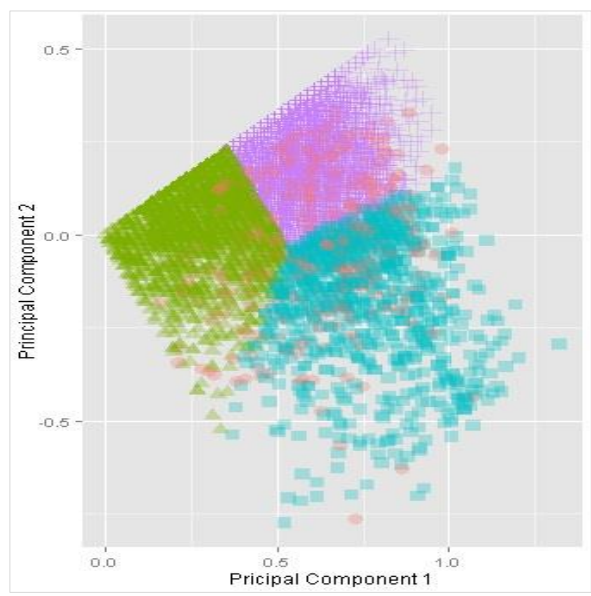

(c) $\mathrm{K}=4$

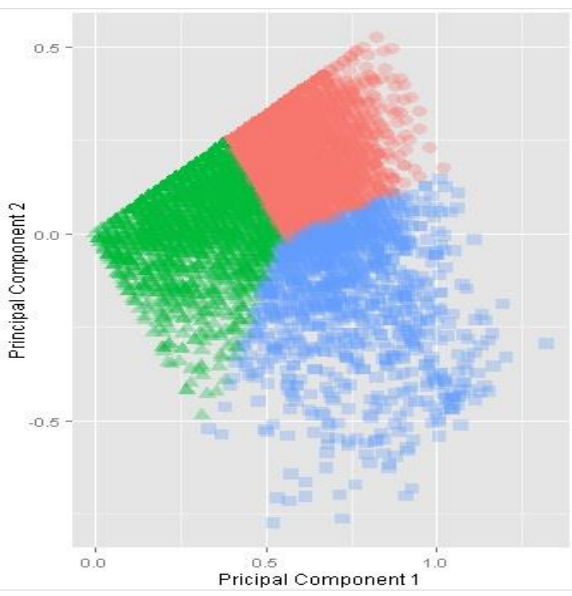

(b) $\mathrm{K}=3$

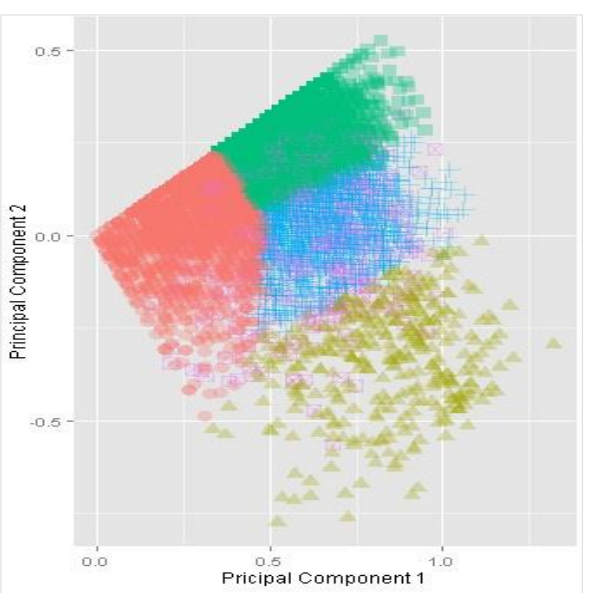

(d) $\mathrm{K}=5$

Figure 4: Visualisation of clustering experiments with K ranging from 2 to 5.

Table 1: Summary of cluster characteristics.

\begin{tabular}{|l|c|c|c|c|c|c|}
\hline & \multirow{2}{*}{$\begin{array}{c}\text { \% o of } \\
\text { Dataset }\end{array}$} & \multicolumn{2}{|c|}{ Gender Distribution } & \multirow{2}{*}{$\begin{array}{c}\text { Avg. } \\
\text { AMales }\end{array}$} & $\begin{array}{c}\text { \%Fg. LOS } \\
\text { (Days) }\end{array}$ & $\begin{array}{c}\text { Avg. TTS } \\
\text { (Days) }\end{array}$ \\
\hline Cluster1 & $45.5 \%$ & $23.4 \%$ & $76.6 \%$ & 86.78 & 8.65 & 1.62 \\
\hline Cluster2 & $18.5 \%$ & $32.7 \%$ & $67.3 \%$ & 83.39 & 31.27 & 2.32 \\
\hline Cluster3 & $36 \%$ & $33.3 \%$ & $66.7 \%$ & 71.67 & 8.57 & 1.62 \\
\hline
\end{tabular}




\subsubsection{Learning Insights from Clusters}

Initially, the clusters were inspected with respect to the potential impact of TTS on LOS. The inpatient LOS is of significant concern in healthcare as a measure of care outcomes (Englert et al. 2001). From an operational standpoint, the LOS was considered as a valid proxy to measure the consumption of hospital resources (Faddy and McClean 1999). Further, it was also reported that the LOS largely accounts for the cost of hip fracture care (Johansen et al. 2013).

Exploring clusters revealed that the patients of Cluster2 tended to have a relatively wider dispersion of the TTS compared to Cluster1 and Cluster3. Interestingly, Cluster2 patients also experienced longer LOS compared to Cluster1 and Cluster3, which shared a very similar distribution of the TTS and LOS variables. Figure 5(a) plots the TTS variable in the three clusters, while Figure 5(b) plots the LOS variable.

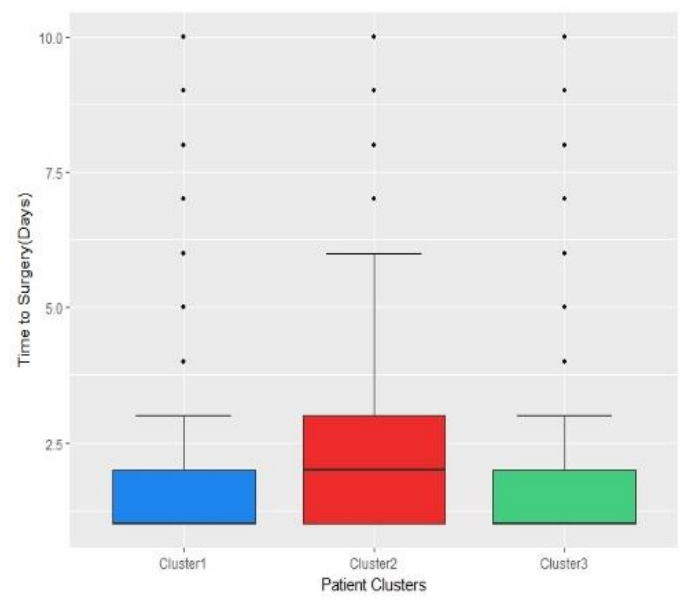

(a) TTS

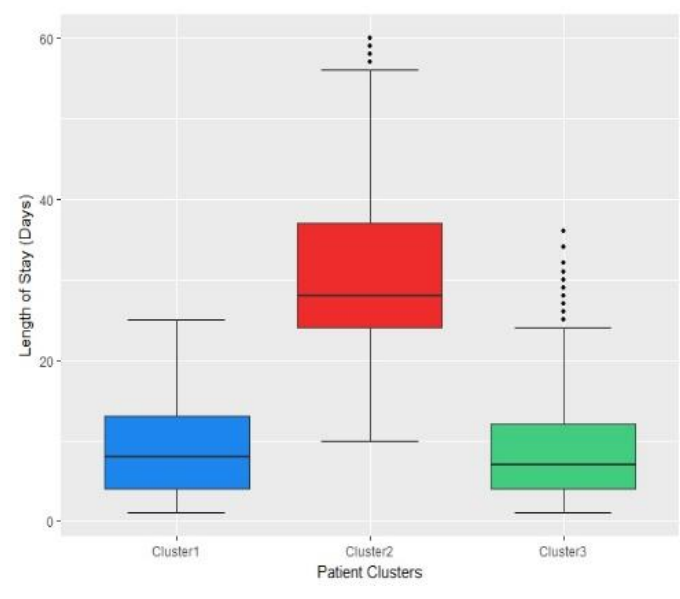

(b) LOS

Figure 5: The variation of the TTS and LOS variables within the three patient clusters.

Subsequently, the age structure of clusters was explored. Generally, there is a strong emphasis on the patient age in elderly care schemes. In this respect, the possibility of sustaining hip fractures is expected to increase significantly with age (Melton 1996). Figure 6 plots the variations of age with respect to the three clusters. On one hand, it can be observed that only Cluster1 and Cluster 2 included all the patients aged 80 or over, while Cluster 3 contained only patients aged less than 80 years.

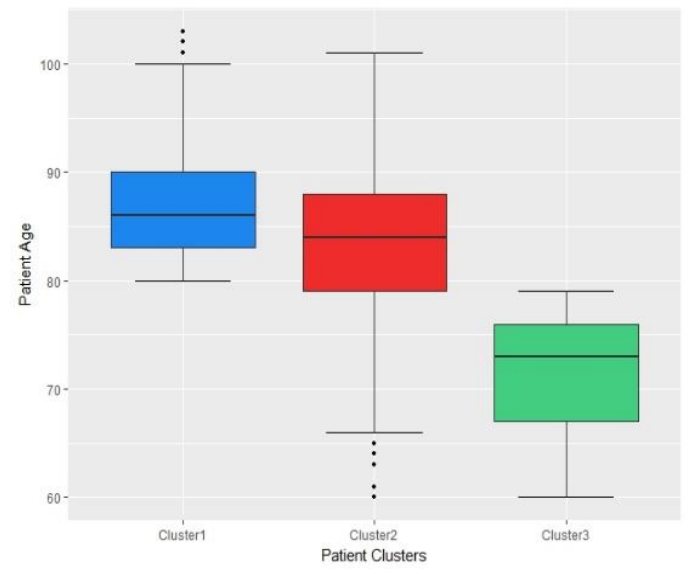

Figure 6: The variation of age in clusters. 


\subsection{Care Pathway Modeling}

\subsubsection{Population-Level Model: Integrating Simulation with ML Insights}

We now demonstrate how the knowledge learned from the ML experiments can be utilized within modeling the healthcare process. The data-driven knowledge was used to reflect on the structure and behavior of the care journey model in different respects as follows.

Initially, a System Dynamics (SD) model was built representing the three clusters of patients. In particular, the model was disaggregated into three stocks corresponding to the clusters of patients. Furthermore, the auxiliary variables were decided based on the cluster analysis conducted before. For instance, the first and second clusters were set to undergo the same TTS delay (i.e. TimeToSurgery1), while the third cluster was assigned a different delay (i.e. TimeToSurgery2).

Similarly, the inflow of elderly patients was structured based on the age variation within clusters. Both of the first and second patient clusters were modelled to include more elderly patients (i.e. aged 80-100), while the third cluster was associated with less elderly patients (i.e. aged 60-80). This reflected the age groups within the patient clusters. In general, the SD model can be used to provide projections of hipfracture patients discharged with a focus on different patient characteristics, and care-related outcomes. Figure 7 illustrates the cluster-based SD model.

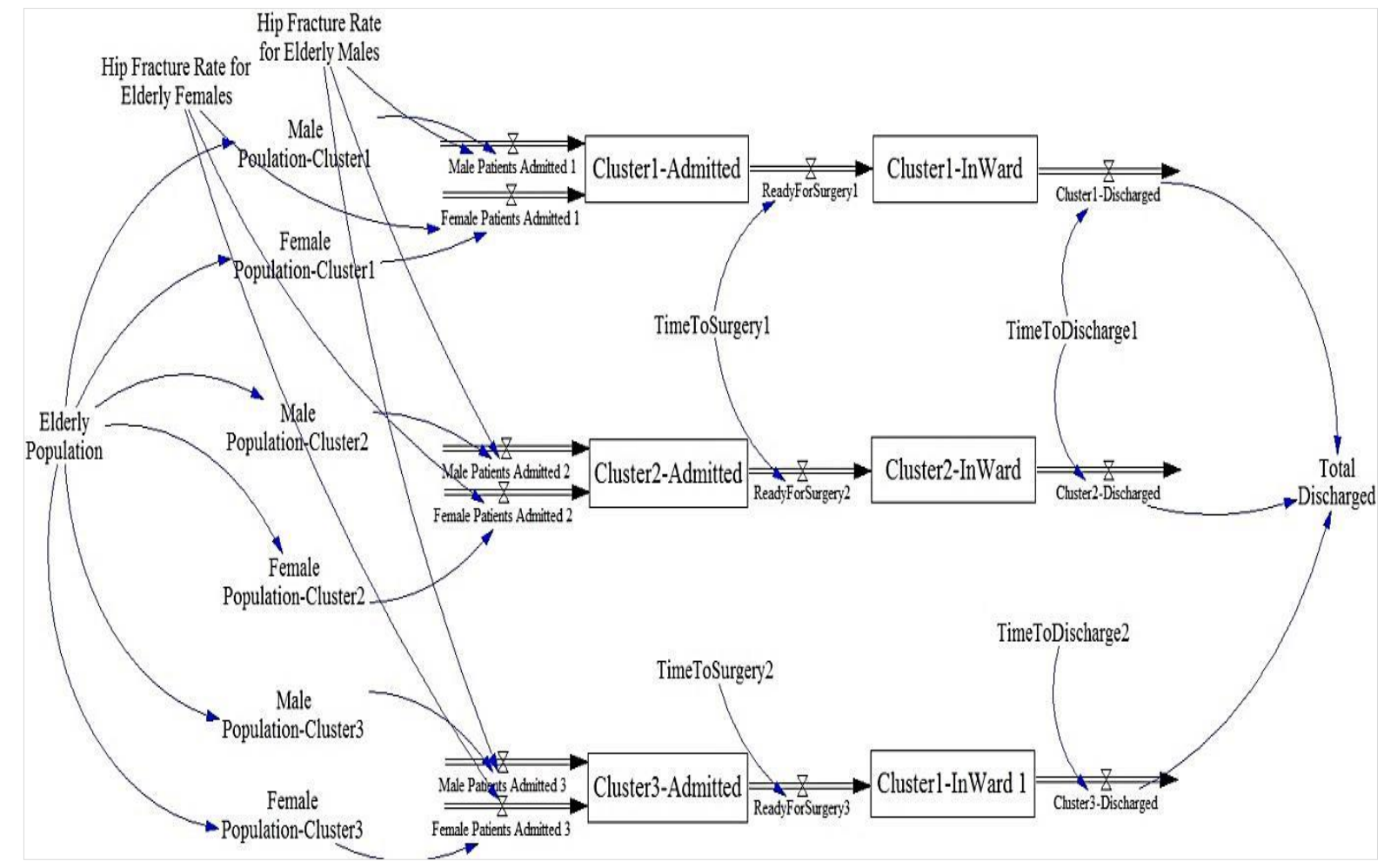

Figure 7: Cluster-based SD model.

\subsubsection{Modeling Care Coordination at Patient-Level}

Subsequently, we model a finer-grained perspective of the care journey while considering patients as entities, rather than aggregate populations. In this manner, each patient could be treated individually in terms of characteristics (e.g. age, gender, type of fracture etc.), and care-related factors (e.g. TTA, TTS etc.). The discrete-event or agent-based modeling would be more suitable in this regard. The model can also be used to produce a realistic sequence of events within the care journey, as sketched in Figure 8. 
Along with the patient journey, points of care coordination are simulated where a change of the process can take effect. Specifically, Figure 8 highlights two components as (Coordination of Care) and (Evaluation). The coordination component initially aims at care intervention including identifying population at risk, implementing early assessment tests as a pre-emptive strategy for reducing the number of admissions. For patient cases, the coordination focuses on the implementation of care standards towards reducing the TTS to be within $48 \mathrm{hrs}$ from admission. The cost of coordination and the improvement in outcomes are reported to the Evaluation component where the ROI is eventually computed.

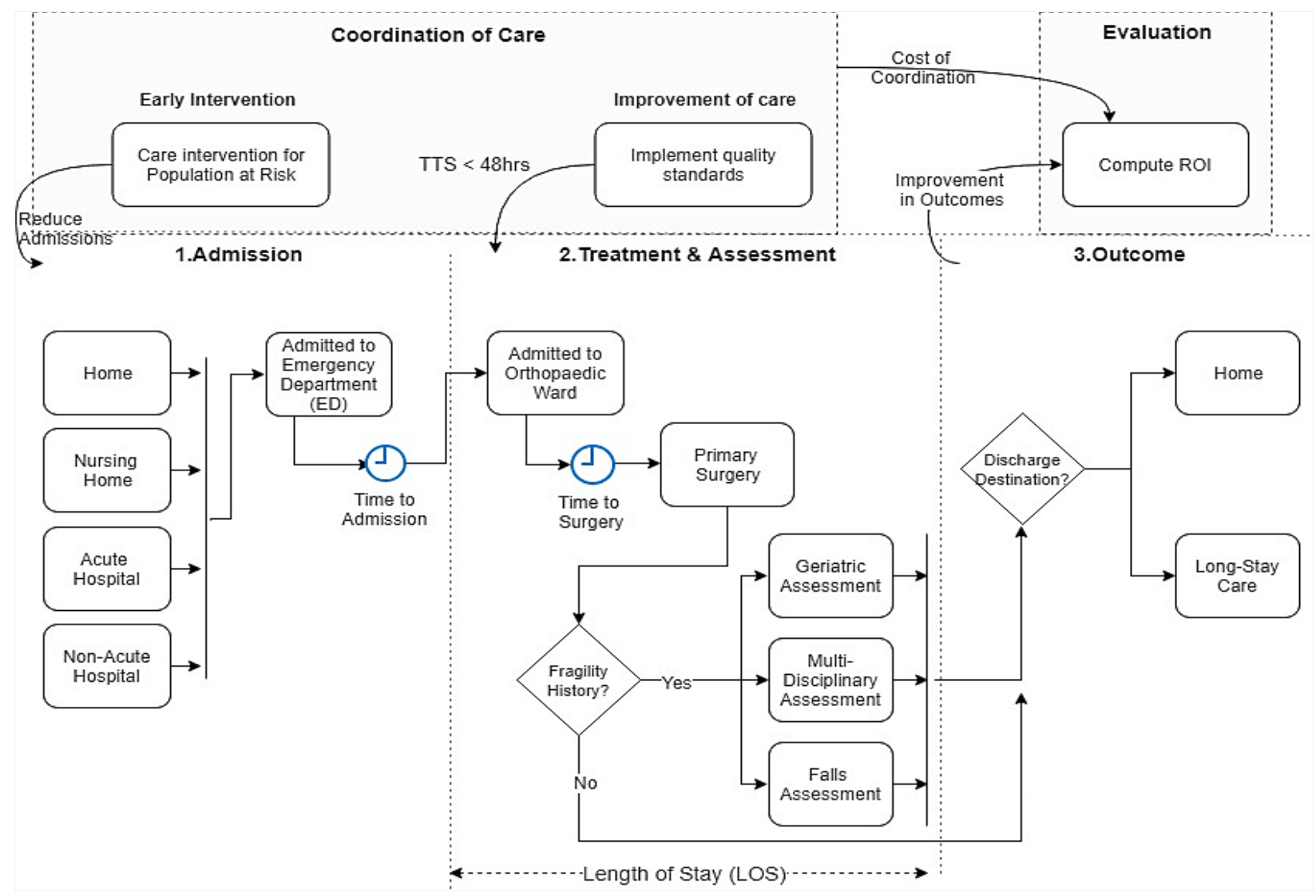

Figure 8: The patient-level model.

\section{CLOSING THOUGHT}

Corollary 2 The key challenges facing healthcare providers in future years are perhaps more organisational and logistical than medical and scientific advances. (Sally Brailsford and Jan Vissers 2011)

We deeply believe in that viewpoint stated above. The healthcare arena can substantially be transformed by advances in our capabilities to understand and model healthcare systems. M\&S presents as a pragmatic method for understanding the complex dynamics underlying healthcare systems towards devising policies for care improvement. It is hoped for the framework presented in this paper to contribute in that direction within the context of developing care pathways.

Further, the paper touched on a methodological prospect by integrating M\&S with ML. The study suggested that ML can be utilised as an assistive artefact within the process of conceptual modeling. Through a practical example, it was demonstrated how insights learned from data clustering could be used effectively to conceptualise the system's structure or behaviour. In a broader context, we conceive that ML can play a key role in tandem with M\&S to help address more complex sets of analytical questions. 


\section{REFERENCES}

Aldenderfer, M.S. and R.K Blashfield. 1984. Cluster Analysis. Sage University Paper Series on Quantitative Applications in the Social Sciences, No.44, Beverly Hills: Sage Publications.

Brailsford, S., and J. Vissers. 2011. "OR in Healthcare: A European Perspective". European Journal of Operational Research 212(2):223-234.

Darowski, A. 2007. The Care of Patients with Fragility Fracture. London: British Orthopaedic Association.

Cooper, C., G. Campion, and L.J. Melton. 1992. "Hip Fractures in the Elderly: A World-Wide Projection". Osteoporosis International, 2(6):285-289.

De Bleser, L., R. Depreitere, K.D. Waele, K. Vanhaecht, J. Vlayen, and W. Sermeus. 2006. "Defining Pathways". Journal of Nursing Management 14(7):553-563.

Dodds, M. K., M. B. Codd, A. Looney, and K. J. Mulhall. 2009. "Incidence of Hip Fracture in the Republic of Ireland and Future Projections: A Population-Based Study". Osteoporosis International 20(12): 2105-2110.

Englert, J., K. M. Davis, and K. E. Koch. 2001. “eUsing Clinical Practice Analysis to Improve Care”. The Joint Commission Journal on Quality Improvement, 27(6):291-301.

EPA. 2018. Retrieved from http://www.e-p-a.org.

Every, N.R., J. Hochman, R. Becker, S. Kopecky, and C.P. Cannon. 2000. "Critical Pathways: A Review". Circulation 101(4):461-465.

Faddy, M. J., and S. I. McClean. 1999. "Analysing Data on Lengths of Stay of Hospital Patients Using Phase-type Distributions". Applied Stochastic Models in Business and Industry 15(4):311-317.

Gold, J. 2015. "Accountable Care Organizations Explained”. Kaiser Health News.

Gröne, O., and M. Garcia-Barbero. 2001. "Integrated Care”. International Journal of Integrated Care, 1(2).

HIPE. 2015. Retrieved from

http://www.hpo.ie/hipe/hipe_data_dictionary/HIPE_Data_Dictionary_2015_V7.0.pdf

HSE. 2008a. Retrieved from $\bar{h}$ ttp://www.lenus.ie/hse/bitstream/10147/46105/4/HSEStrategyFalls.pdf. Health Service Executive (HSE).

HSE. 2008b. "Strategy to Prevent Falls and Fractures in Ireland's Ageing Population Summary, Conclusions and Recommendations". Health Service Executive (HSE).

HSE. 2014. Annual Report and Financial Statements 2014. Health Service Executive (HSE)

HSE. 2015. Retrieved from http://www.hse.ie/eng/services/publications/corporate/CHO_Chapter_1.pdf.

Husni, M.E., E. Losina, A.H. Fossel, D.H. Solomon, N.N. Mahomed, and J.N. Katz. 2010 . "Decreasing Medical Complications for Total Knee Arthroplasty: Effect of Critical Pathways on Outcomes". BMC Musculoskeletal Disorders 11(1):160.

Johansen, A., R. Wakeman, C. Boulton, F. Plant, J. Roberts, and A. Williams. 2013. "National Hip Fracture Database: National Report 2013”. London: Royal College of Physicians.

Law, A. M. 2007. Simulation Modeling \& Analysis. 4th ed. Singapore: McGraw-Hill Education.

Melton, L. J. 1996. "Epidemiology of Hip Fractures: Implications of the Exponential Increase with Age". Bone, 18(3):121-125.

Pearson, S.D., D. Goulart-Fisher, and T.H. Lee. 1995. "Critical Pathways as a Strategy for Improving Care: Problems and Potential". Annals of Internal Medicine, 123(12):941-948.

Pidd, M. 2004. "Complementarity in Systems Modelling". In Systems Modelling: Theory and Practice, edited by M. Pidd Chichester, 1-20. England: John Wiley \& Sons.

Rotter, T., L. Kinsman, E.L. James, A. Machotta, H. Gothe, J. Willis, P. Snow, and J. Kugler. 2010. "Clinical Pathways: Effects on Professional Practice, Patient Outcomes, Length of Stay and Hospital Costs". Cochrane of Systematic Reviews (3): CD006632-1.

Royston, P., G. Ambler, and W. Sauerbrei. 1999. "The Use of Fractional Polynomials to Model Continuous Risk Variables in Epidemiology”. International Journal of Epidemiology 28(5):964-974.

Shearer, C. 2000. "The CRISP-DM Model: The New Blueprint for Data Mining". Journal of Data Warehousing 5(4):13-22. 
Shi, J., Q. Su, and Z. Zhao. 2008. "Critical Factors for the Effectiveness of Clinical Pathway in Improving Care Outcomes". In Proceedings of the International Conference on Service Systems and Service Management, $30^{\text {th }}$ June- $2^{\text {nd }}$ July, Melbourne, Australia, 1-6.

Vanhaecht, K. 2007. The Impact of Clinical Pathways on the Organization of Care Processes. PhD Thesis, KU Leuven. https://irias.kuleuven.be/retrieve/92842.

Vanhaecht, K., M. Panella, R. Van Zelm, and W. Sermeus. 2010. "An Overview on the History and Concept of Care Pathways as Complex Interventions”. International Journal of Care Pathways 14(3):117-123.

Wanless, D. 2004. Securing Good Health for the Whole Population. London: HM Treasury.

Zeigler, B.P., and H.S. Sarjoughian. 2013. Guide to Modeling and Simulation of Systems of Systems (Simulation Foundations, Methods and Applications). 2nd ed. London: Springer.

Zeigler, B. P., E. L. Carter, O. Molloy, and M. Elbattah. 2016. "Using Simulation Modeling to Design Value-Based Healthcare Systems". In Proceedings of the Operational Research Society Annual Conference (OR58), $6^{\text {th }}-8^{\text {th }}$ September, Portsmouth, England.

Zeigler, B.P., 2016. "Discrete Event System Specification Framework for Self-Improving Healthcare Service Systems”. IEEE Systems Journal (99):1-12.

\section{AUTHOR BIOGRAPHIES}

MAHMOUD ELBATTAH is a post-doctoral researcher at the MIS Lab (Laboratoire Modélisation, Information, Systèmes) based in Université de Picardie Jules Verne, France. He received his $\mathrm{PhD}$ in Informatics from the National University of Ireland Galway. His research interests include Simulation Modeling and Machine Learning with a focus on applications in healthcare. His e-mail address is mahmoud.elbattah@u-picardie.fr.

OWEN MOLLOY is a Lecturer in Information Technology at the National University of Ireland Galway. His research interests are in the areas of business process modeling and simulation, software engineering, healthcare processes, and information systems. His e-mail address is owen.molloy@nuigalway.ie.

BERNARD P. ZEIGLER is Chief Scientist at RTSync Corp., Professor Emeritus of Electrical and Computer Engineering at the University of Arizona (UA) and Affiliated Research Professor in the C4I Center at George Mason University. He is internationally known for his seminal contributions in modeling and simulation theory. He has published several highly cited books including "Theory of Modeling and Simulation" (3rd edition in process) and "Guide to Modeling and Simulation of Systems of Systems." He is the originator of the Discrete Event System Specification (DEVS) formalism in 1976 which has spurred the development of a world-wide research community, with its own conference meetings, and research/technology awards. In 1995, Zeigler was named Fellow of the IEEE in recognition of his contributions to the theory of discrete event simulation. Dr. Zeigler co-founded the Arizona Center for Integrative Modeling and Simulation (ACIMS) in 2001 dedicated to the development of modeling and simulation as a discipline of the future. Dr. Zeigler has been the lead architect of multiple web-based systems in complex systems that incorporate unique behaviors derived from DEVS-based properties. He is also Fellow of the Society for Modeling and Simulation International and member of its Hall of Fame. He received the Life-time Achievement Award from INFORMS in 2016. His email address is zeigler@ece.arizona.edu. 